\title{
Digital direct electron imaging of energy-filtered electron backscatter diffraction patterns
}

\author{
S. Vespucci, ${ }^{1}$ A. Winkelmann, ${ }^{2}$ G. Naresh-Kumar, ${ }^{1}$ K. P. Mingard, ${ }^{3}$ D. Maneuski, ${ }^{4}$ P. R. Edwards, ${ }^{1}$ A. P. Day, ${ }^{5}$ \\ V. O'Shea, ${ }^{4}$ and C. Trager-Cowan ${ }^{1, *}$ \\ ${ }^{1}$ Department of Physics, SUPA, University of Strathclyde, Glasgow G4 ONG, United Kingdom \\ ${ }^{2}$ Bruker-Nano, Am Studio 2D, 12489 Berlin, Germany \\ ${ }^{3}$ National Physical Laboratory, Teddington, Middlesex TW11 OLW, United Kingdom \\ ${ }^{4}$ School of Physics and Astronomy, SUPA, University of Glasgow, Glasgow G12 8QQ, United Kingdom \\ ${ }^{5}$ Aunt Daisy Scientific Ltd, Claremont House, High Street, Lydney GL15 5DX, United Kingdom \\ (Received 13 March 2015; revised manuscript received 26 June 2015; published 6 November 2015)
}

\begin{abstract}
Electron backscatter diffraction is a scanning electron microscopy technique used to obtain crystallographic information on materials. It allows the nondestructive mapping of crystal structure, texture, and strain with a lateral and depth resolution on the order of tens of nanometers. Electron backscatter diffraction patterns (EBSPs) are presently acquired using a detector comprising a scintillator coupled to a digital camera, and the crystallographic information obtainable is limited by the conversion of electrons to photons and then back to electrons again. In this article we will report the direct acquisition of energy-filtered EBSPs using a digital complementary metal-oxide-semiconductor hybrid pixel detector, Timepix. We show results from a range of samples with different mass and density, namely diamond, silicon, and GaN. Direct electron detection allows the acquisition of EBSPs at lower $(\leqslant 5 \mathrm{keV})$ electron beam energies. This results in a reduction in the depth and lateral extension of the volume of the specimen contributing to the pattern and will lead to a significant improvement in lateral and depth resolution. Direct electron detection together with energy filtering (electrons having energy below a specific value are excluded) also leads to an improvement in spatial resolution but in addition provides an unprecedented increase in the detail in the acquired EBSPs. An increase in contrast and higher-order diffraction features are observed. In addition, excess-deficiency effects appear to be suppressed on energy filtering. This allows the fundamental physics of pattern formation to be interrogated and will enable a step change in the use of electron backscatter diffraction (EBSD) for crystal phase identification and the mapping of strain. The enhancement in the contrast in high-pass energy-filtered EBSD patterns is found to be stronger for lighter, less dense materials. The improved contrast for such materials will enable the application of the EBSD technique to be expanded to materials for which conventional EBSD analysis is not presently practicable.
\end{abstract}

DOI: 10.1103/PhysRevB.92.205301

PACS number(s): 61.05.J-

\section{INTRODUCTION}

In the development and study of new materials, the understanding of their crystal structure plays a crucial rule. Electron backscatter diffraction (EBSD), also known as backscatter Kikuchi diffraction (BKD), is a technique used to obtain accurate crystallographic information from bulk materials, thin films, and nanoparticles with high spatial resolution [1] (of order $20 \mathrm{~nm}$ ). Typical materials which can be investigated by EBSD include metals, rocks, ceramics, and semiconductors. The technique allows the identification of individual grain orientations, grain boundaries, and phase identification [1,2]. It is also used to study processes such as recrystallization and grain growth, and it is a very powerful tool in the study of strain fields in crystals [3].

Experimentally, EBSD is conducted in a scanning electron microscope (SEM) equipped with an EBSD detector. Commercial EBSD detectors consist of an electron-sensitive screen (a phosphor or a scintillator) placed in front of the

*c.trager-cowan@strath.ac.uk

Published by the American Physical Society under the terms of the Creative Commons Attribution 3.0 License. Further distribution of this work must maintain attribution to the author(s) and the published article's title, journal citation, and DOI. specimen whose normal is usually tilted by approximately $70^{\circ}$ with respect to the exciting electron beam. A digital camera is used to acquire an image of the diffraction pattern formed by the backscattered electrons impinging on the screen $[1,4]$; this is illustrated in Fig. 1(a). The use of the phosphor screen introduces a detrimental conversion of electrons to photons, by the phosphor, and from photons back to electrons by the charge-coupled device (CCD) camera. There are intrinsic light scattering and optical absorption within the phosphor which introduce an intrinsic loss of spatial resolution. This is a limiting factor in applications where it is required to measure small changes in the patterns, for example, in strain measurement [3,5], or where the change in the contrast and sharpness can provide information on the specimen crystalline quality $[3,6]$.

An electron backscatter diffraction pattern is shown in Fig. 1(b). A detailed description of the intensities in the electron backscatter diffraction patterns (EBSPs) is possible using a Bloch wave approach to the dynamical theory of electron diffraction $[7,6]$; however, the geometry of the EBSP can be described, to a first approximation, by considering the angular position of electrons which have been Bragg reflected from the lattice planes of the crystal specimen. On penetrating the specimen, the electrons of the impinging beam are both elastically and inelastically scattered. This creates a diverging source of electrons with a broad range of energies [2,8-14]. Some of those electrons travel in such a way that their energy 


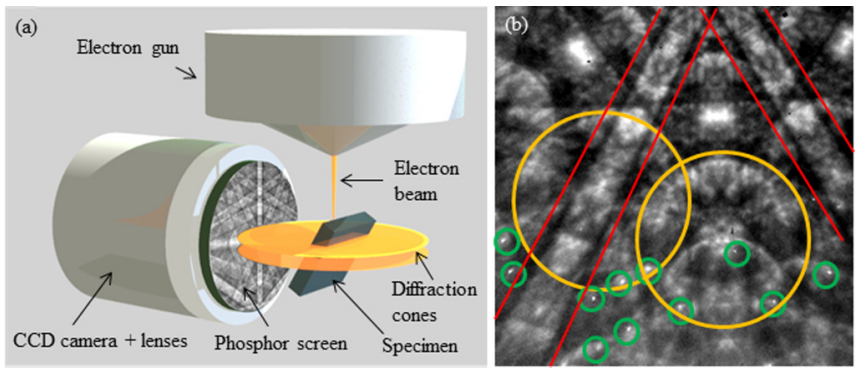

FIG. 1. (Color online) (a) Illustration of the EBSD detection geometry and a conventional EBSD detector. (b) An EBSP from a GaN thin film acquired at an energy of $5 \mathrm{keV}$, a probe current of $\approx 1.5 \mathrm{nA}$, a detector-to-specimen distance of $\approx 10 \mathrm{~mm}$ (capture angle $\approx 60^{\circ}$ ), and a $100 \mathrm{~s}$ exposure time using the Timepix digital direct electron imaging detector. The red lines outline a pair of Kikuchi bands; the large yellow circles indicate HOLZ rings and the small green circles highlight RHEED spots.

and direction satisfy the Bragg condition, $2 d \sin \theta=n \lambda$, for a set of planes and undergo diffraction, where $d$ is the spacing between planes, $\theta$ is the Bragg angle, $\lambda$ the wavelength of the electron, and $n$ the order of diffraction. Because of the cylindrical symmetry of the Bragg reflection condition with respect to the lattice plane normal, diffraction cones (Kossel cones) are formed. When these cones intersect the phosphor screen, Kikuchi lines are observed in a gnomonic projection; see Fig. 1. The Kikuchi lines appear as almost straight lines because the cones are very shallow, as the Bragg angle $\theta$ is of order $1^{\circ}$. As each Kikuchi band (pair of Kikuchi lines) is effectively fixed to the plane from which it is formed, an EBSD pattern provides a direct measurement of a sample's orientation. Rotation of a crystal will produce a rotation of the EBSP; a tilt of a crystal will produce a shift in the EBSP. EBSPs acquired from a mesh of points on a sample can be used to produce a map of tilts or rotations in that sample [1].

The Kikuchi bands are the main features used to extract information from EBSPs in commercial systems, but circlelike features called high-order Laue zone (HOLZ) rings are also observed [4,6]; see Fig. 1(b). The fine structure in HOLZ rings is very energy and lattice-parameter dependent; if these could be recorded with sufficient detail, this could in principle allow the determination of the lattice parameters with very high precision $[6,15,16]$. Reflection high-energy electron diffraction (RHEED) spots may also appear in the EBSP due to glancing angle coherent scattering of the incident beam. They are visible in the EBSP in Fig. 1(b). RHEED patterns may be used to determine a sample's surface reconstruction and may also provide a precise measure of the lattice constant $[17,18]$.

There is ongoing research to produce a detailed understanding of the physical mechanisms involved in the formation of the diffraction patterns. By using the dynamical theory of diffraction approach it is possible to obtain EBSP simulations showing good agreement with the experimental patterns $[7,19,20]$. However, the range of energies of the electrons contributing to the EBSP and the depth from which they emerge from the sample is still not completely understood $[6,8,15,21]$.

To interrogate the energies of the electrons contributing to the EBSPs, energy-resolved EBSPs have been recorded using an electrostatic energy filter placed between the sample and the phosphor screen $[22,23]$, or diffraction band contrast has been measured as function of the electron energy loss [8,24]. An increase in the contrast and sharpness of diffraction features is observed when only electrons having energy close to the primary beam are detected, indicating that the largest contribution to EBSP formation is made by low-loss electrons. High-loss and thus lower-energy electrons appear to give rise predominantly to a featureless background whose effect is a reduction of the diffraction contrast and sharpness in the diffraction pattern.

We can roughly differentiate between three groups of scattered electrons:

(1) Elastically scattered electrons which are either

(1a) coherently elastically scattered (e.g., RHEED spots) or

(1b) incoherently quasielastically scattered (through phonon scattering-energy loss typically less than $1 \mathrm{eV}$ ).

The number of group (1a) electrons is usually much smaller than group (1b) electrons for a typical EBSD experiment.

(2) Inelastically scattered electrons which have lost only a relatively small amount of energy of the order of typically a few plasmon energies (tens of eV).

(3) Inelastically scattered electrons which have lost significant energy (several $100 \mathrm{eV}$ ). Diffraction effects are exhibited by groups (1) and (2), comprising the low-loss electrons plus elastically scattered electrons. The diffraction effects are gradually diminished for electrons with increasing energy loss in group (2). For more detailed discussion see Refs. [21,23].

In the present work we propose a method for the acquisition of energy-filtered EBSPs where direct electron detection and energy filtering is achieved using a digital complementary metal-oxide semiconductor (CMOS) hybrid pixel detector, Timepix [25]. This approach avoids the use of the phosphor screen and CCD camera combination, and allows energy filtering to be accomplished without any additional hardware: the functionality is implemented in the electronics chip.

The advantages of this system include the enabling of a reduction in the electron beam energy, beam current, and acquisition time compared to conventional systems, noiseless acquisition, and most importantly, energy discrimination. This allows the acquisition of small-scale details in the EBSP which are not in practice obtainable with existing commercial EBSD systems. This may provide routes to, for example, the determination of lattice constant, crystal phase identification, and the mapping of strain with greater sensitivity $[6,15]$.

Wilkinson et al. [26] have previously demonstrated the advantages of using direct electron imaging for the acquisition of EBSPs. They used a directly exposed (CMOS) sensor and demonstrated that higher-quality patterns may be acquired compared to those recorded using conventional indirect detectors, particularly at low voltages. Our work illustrates the additional advantages of applying energy filtering. 


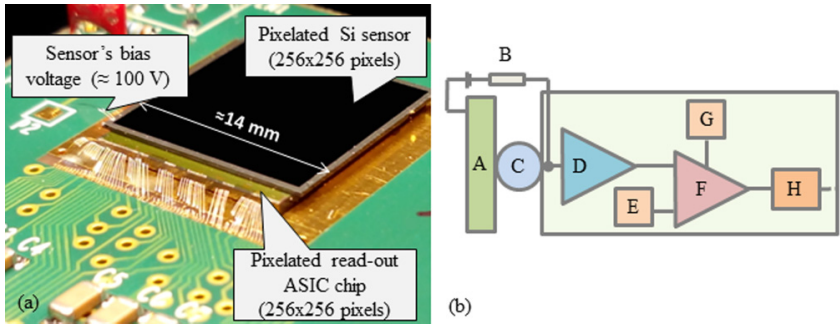

FIG. 2. (Color online) (a) Timepix detector, (b) simplified schematic of a detector's pixel: sensor (A), bias voltage (B), solder bumps $(\mathrm{C})$, preamplifier $(\mathrm{D})$, threshold $(\mathrm{E})$, discriminator $(\mathrm{F})$, threshold adjustment $(\mathrm{G})$, and counter $(\mathrm{H})$.

\section{A DIGITAL CMOS HYBRID PIXEL DETECTOR-TIMEPIX}

In the last few decades considerable progress has been made in the field of CMOS technology. It is now possible to pack a very large number of components into a very small area, allowing the creation of an application-specific integrated circuit (ASIC), consisting of integrated circuits customized and optimized for a specific rather than general purpose use [27].

Timepix is one of the outcomes of an international collaboration (Medipix2) hosted at CERN, established to provide a solution for a range of problems in X-ray and gamma-ray imaging [28]. The Timepix detector belongs to the CMOS hybrid pixel detectors family [25,28,29]. The Timepix chip was derived directly from the Medipix 2 chip development. It shares most of the properties of the Medipix 2 chip, but has additional functionality, in that with the Timepix chip it is possible to obtain timing information (time over threshold and time of arrival). Hybrid pixel detectors such as Timepix can be regarded as digital direct electron detectors. They count events rather than give an output proportional to a fraction of the total energy of the detected particle, as in the case of widely used analog detectors such as CCDs or monolithic active pixel sensors. In the latter case there is variability in the output depending on the path traveled by a detected particle inside the sensor, and there is always intrinsic noise in the device, which reduces the signal-to-noise ratio [27,30].

In the case of a hybrid pixel detector, the sensor and the electronics chip are independent, offering the possibility of the choice of sensor material and sensor thickness depending on the specific application; in the present case the sensor comprises $300 \mu \mathrm{m}$ of silicon. The detector consists of arrays of individual independent counters. Each pixel of the sensor has its own amplification pixel in the electronics chip. Sensor and electronics are composed of $256 \times 256$ square pixels, each $55 \mu \mathrm{m} \times 55 \mu \mathrm{m}$ in size, covering a global surface of around $1.4 \mathrm{~cm} \times 1.4 \mathrm{~cm}$ [Fig. 2(a)]; it is also possible to combine chips to obtain a bigger sensor area. Each pixel of the sensor is electrically and mechanically connected, through solder bumps, to the corresponding pixel of the CMOS ASIC electronics chip [25,27].

Each pixel of the electronics is basically composed [see Fig. 2(b)] of a charge sensitive amplifier, a discriminator, and a 14-bit counter [25]. The basic operating principle is as follows: when a particle hits a sensor pixel, a cloud of charges (electron-hole pairs) is generated within the material of the sensor. The quantity of generated charge is proportional to the total energy deposited within the sensor by the particle. The charges, drifting under the effect of the electric field applied to the sensor, are collected by the solder bump and transferred to the ASIC electronic chip [25]. The signal from the sensor is amplified by the shaping preamplifier and then compared, by the discriminator, with a threshold value. If the signal is greater than the specified threshold value, the discriminator generates a logic signal whose width is proportional to the time for which the voltage at the output of the preamplifier is above the threshold. The global threshold set for the chip can be adjusted individually for each pixel, in order to compensate for small differences between pixels. An equalization of the matrix is typically performed in order to have a more uniform response within the global detector area. If the threshold level is set above the intrinsic noise of the device, it is possible to operate in noise-free conditions [25].

The digital output from the discriminator is processed depending on the acquisition mode of the device. Each pixel of the detector can be individually programmed in order to work in one of the three possible configurations: Medipix, Time Over Threshold, and Time Of Arrival mode [25]. In this article we will describe experimental results from the detector working in Medipix mode. In this mode the device is used as a counter. For each pixel, the internal counter increments one unit every time an electron has energy above the threshold value. Images are recorded using an exposure which does not overflow the maximum pixel counter range (11810 counts per frame), but acquisition in integral mode allows acquisition with virtually infinite dynamic range. The whole chip is read out through a serial (or parallel) interface [25,27]. In our case, the readout operation and data acquisition are managed by a FitPix readout interface and Pixelman software [31-33].

Because in a hybrid pixel technology the sensor is separate from the electronics, there is negligible radiation damage from the incident particle if the energy of the particle is below a specific value [30]. In the range of energies available in a SEM, from 0 to $30 \mathrm{keV}$, we believe we can completely ignore the problem of radiation damage.

The imaging properties of the detector have been extensively studied [30,34]. Parameters used to define the performance of an imaging detector are the modulation transfer function (MTF), which gives a measure of the spatial frequency response of a detector, and the noise power spectrum (NPS), which describes the spectral component of the noise added to the image by the detection system. These two quantities may be combined to obtain the detective quantum efficiency (DQE). A characteristic value of the DQE is the one at zero spatial frequency, which is referred to as the effective quantum efficiency of the system-see Refs. [30,34] for a detailed description of these parameters.

For detectors such as the Timepix and Medipix2, the MTF, NPS, and DQE vary as a function of the applied threshold level $[30,34]$. When a particle hits the border of a pixel, it can share its energy between pixels. In this case, if the threshold level is high enough, the energy deposited in a given pixel is not sufficiently high to trigger the pixel count. This results in a reduction of the effective active pixel area; that is, the peripheries of the pixels are effectively not active. For this reason, as the threshold is increased, there is a consequent 

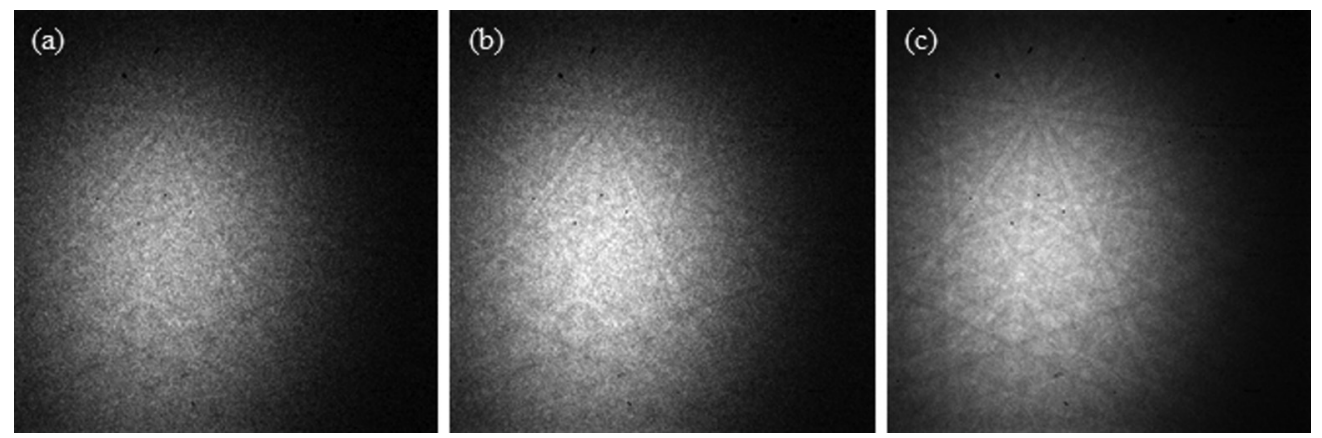

FIG. 3. As-acquired EBSPs from GaN for an incident beam energy of $20 \mathrm{keV}$ with a threshold energy of $19.4 \mathrm{keV}$, a probe current of $\approx 20 \mathrm{nA}$, and a detector-to-specimen distance of $\approx 5 \mathrm{~mm}$ providing a capture angle of $100^{\circ}$, with an acquisition time of (a) $0.5 \mathrm{~ms}$, (b) $1 \mathrm{~ms}$, and (c) $5 \mathrm{~ms}$.

increase in the MTF, which can reach a value larger than the theoretical value of the MTF obtainable using Nyquist's sampling theory for a specific pixel pitch [34]. On the other hand, an increase in the threshold level causes an increase in the NPS, with a consequent reduction in the DQE as DQE $\propto \frac{\mathrm{MTF}^{2}}{\mathrm{NPS}}$.

The properties of the Medipix 2 chip have been characterized by Tlustos et al. in Ref. [34]; the MTF and DQE were measured using the spectrum from a $25-\mathrm{keV} \mathrm{W}$ tube and a $300-\mu$ m-thick silicon sensor. Using a threshold energy of $9.1 \mathrm{keV}$, they measured a DQE of $25 \%-26 \%$, which approaches the maximum theoretical value of $27 \%$ for the detector. Using a threshold energy of $18.5 \mathrm{keV}$, the DQE was found to reduce to $5 \%$. The spatial resolution was found to vary between 8.2 line pairs $/ \mathrm{mm}$ and 11 line pairs $/ \mathrm{mm}$ for a MTF of 70\% for threshold energies between 9.1 and $18.5 \mathrm{keV}$, respectively [34].

All the characteristics of hybrid pixel detectors as presented above make this family of detectors unique with respect to other currently existing technologies. A more detailed description of the detectors and a comparison between the technologies of different detectors and their performance are described in Refs. [27,30].

\section{RESULTS AND DISCUSSION}

EBSPs were acquired in an FEI Sirion Schottky field emission scanning electron microscope operating in spot mode. Our detector was positioned in front of a specimen which was tilted so that the sample normal was $70^{\circ}$ away from the direction of the incident electron beam. Figure 1(b) illustrates the capabilities of the detector in acquiring low-energy EBSPs and shows an EBSP obtained from a single-crystal GaN thin film. This was acquired at an incident electron beam energy of $5 \mathrm{keV}$, a probe current of $\approx 1.5 \mathrm{nA}$, a 100 -s exposure time, and a detector-to-specimen distance of $\approx 10 \mathrm{~mm}$ which provided a capture angle of $\approx 60^{\circ}$. Detectorto-specimen distances and capture angles were determined subsequent to measurement by comparison of the acquired EBSPs with dynamical simulations of the EBSPs produced using the Bruker's ESPRIT DYNAMICS software [35]. To date we have acquired EBSPs at incident electron beam energies down to $3 \mathrm{keV}$. The EBSP shown in Fig. 1(b) has been flat fielded by dividing the as-acquired EBSP by an image acquired on scanning the beam over a large area of the sample. Scanning the beam effectively averages out the diffraction information so no diffraction pattern is observed, thus providing a "background" which can be used to flat field the raw EBSP [1].

Acquisition times depend on beam energy, current, the mass and density of the material under study, and the required quality (signal-to-noise ratio) of the EBSP. To date we have acquired EBSPs with the Timepix detector that were of sufficient quality to allow identification of the Kikuchi bands, with acquisition times down to $0.5 \mathrm{~ms}$. Figure 3 shows examples of as-acquired (i.e., not flat fielded) energy-filtered EBSPs from a singlecrystal GaN thin film recorded with acquisition times of 0.5 , 1 , and $5 \mathrm{~ms}$, respectively. These were obtained at an incident beam energy of $20 \mathrm{keV}$ with a threshold energy of $19.4 \mathrm{keV}$, a beam current of order $20 \mathrm{nA}$, and a detector-to-specimen distance of $\approx 5 \mathrm{~mm}$ providing a capture angle of $\approx 100^{\circ}$. In the EBSP acquired with an acquisition time of $0.5 \mathrm{~ms}$ [Fig. 3(a)], the number of counted electrons is extremely small, even zero at the periphery of the EBSP, due to the angular distribution of the electron intensity. This illustrates that the acquisition time is not limited by the detector but by the available electrons, which is determined by the beam current, and by the angular distribution and energy distribution of backscattered electrons.

To demonstrate the effect of energy filtering, we acquired EBSPs from diamond, $\mathrm{Si}$, and GaN single-crystal films with an incident electron beam energy of $20 \mathrm{keV}$, a probe current of $\approx 10 \mathrm{nA}$, and detector-to-specimen distances of between $\approx 8$ and $15 \mathrm{~mm}$, which provided capture angles of between $\approx 80^{\circ}$ and $50^{\circ}$; these are shown in Fig. 4. These materials were selected to demonstrate the capabilities of the Timepix detector for materials with a range of masses and densities. Flat fielded EBSPs were acquired with the Timepix threshold energy set low (5.5 keV for diamond and GaN and $4.6 \mathrm{keV}$ for $\mathrm{Si}$ ) and set high and close to the incident beam energy (19.4 keV for all three samples). The threshold energies were subsequently determined through energy calibration of the detector threshold obtained by monitoring the signal on the detector for a range of threshold values and electron beam energies incident on a sample.

Figure 5 shows the differential of the intensity (total electron count) of backscattered electrons as a function of the digital, discrete, threshold (THL) value for a range of incident beam energies. The THL value which corresponds 

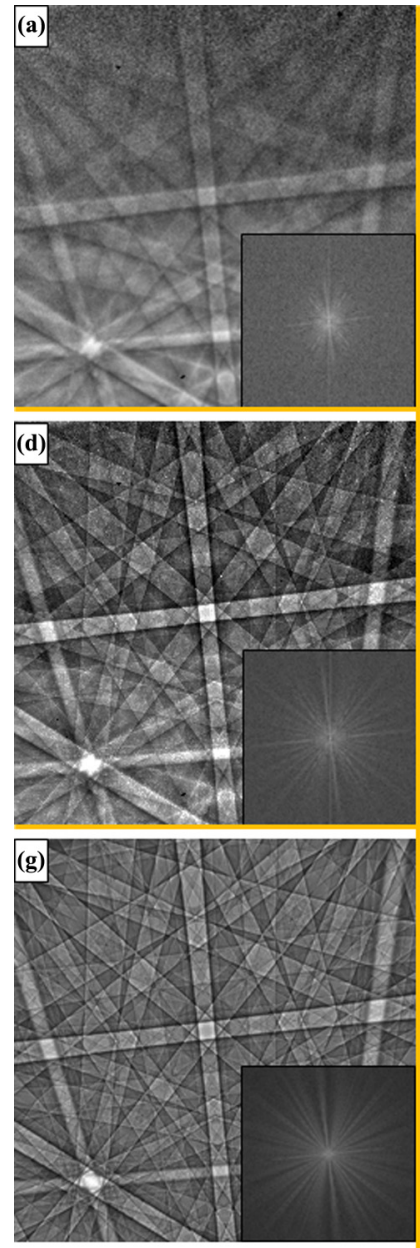

Diamond
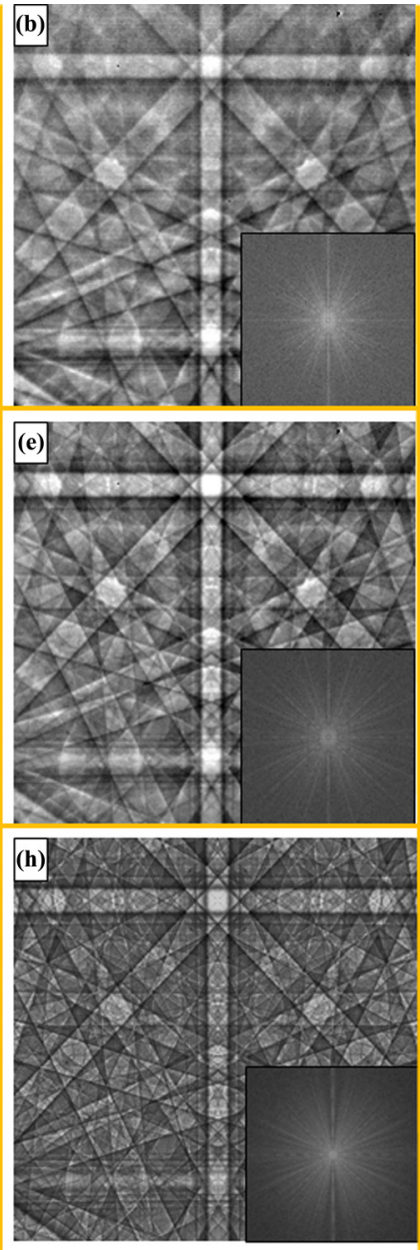

Silicon
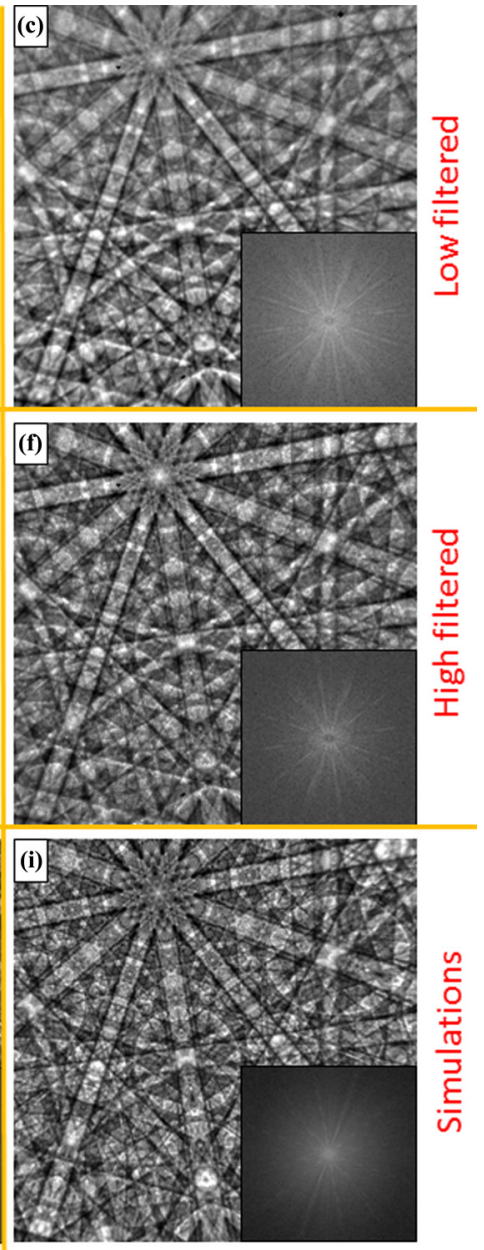

$\mathrm{GaN}$

FIG. 4. (Color online) (a-c) Experimental EBSPs from diamond, Si, and GaN for an incident beam energy of $20 \mathrm{keV}$, a probe current of $\approx 10 \mathrm{nA}$, and a threshold energy of $4.6 \mathrm{keV}$. The EBSPs from diamond, Si, and GaN had acquisition times of $0.8,50$, and $10 \mathrm{~s}$, respectively. The insets are two-dimensional (2D) fast Fourier transforms of each image. (d-f) Experimental EBSPs from diamond, Si, and GaN for an incident beam energy of $20 \mathrm{keV}$, a probe current of $\approx 10 \mathrm{nA}$, and a threshold energy of $19.4 \mathrm{keV}$. The EBSPs from diamond, Si, and GaN had acquisition times of 100, 1482, and $60 \mathrm{~s}$, respectively. The insets are 2D fast Fourier transforms of each image. (g-i) $19.5 \mathrm{keV}$ dynamical simulations of EBSPs from diamond, Si, and GaN. The detector-to-specimen distances and capture angles for the EBSPs were estimated by comparison with the simulated EBSPs. For diamond the detector-to-specimen distance was $\approx 8 \mathrm{~mm}$ with a capture angle $\approx 80^{\circ}$, for Si the detector-to-specimen distance was $\approx 15 \mathrm{~mm}$ with a capture angle $\approx 50^{\circ}$, and for $\mathrm{GaN}$ the detector-to-specimen distance was $\approx 10 \mathrm{~mm}$ with a capture angle $\approx 70^{\circ}$.

to a given energy is estimated by extrapolation of the curves of Fig. 5 to zero intensity. The inset to Fig. 5 shows the resultant calibration curve of electron energy versus THL which exhibits very good linearity.

The minimum practicable increase in the threshold energy corresponds to one unit of the digital THL value, which corresponds to $\approx 90 \mathrm{eV}$ from the slope of the linear fit.

From the energy calibration performed using $\mathrm{x}$ rays by Carramate et al. [36] the energy resolution of the Medipix2 chip has been determined to be of the order of $2 \mathrm{keV}$ [36]. However, Fig. 5 illustrates that it is possible to discriminate between curves of detected intensity versus THL for a small change in the electron beam energy $(\approx 200 \mathrm{eV})$. This implies that while it is not possible to completely resolve energies less than $\approx 2 \mathrm{keV}$ apart, for a given threshold value, electrons with energy less than the corresponding energy are detected with a much lower weight/probability.
The calibration procedure and its comparison with the conventional method (using radioactive sources) will be described in detail in Ref. [37].

For the EBSPs presented in this paper, acquisition times were set significantly higher to provide very high quality patterns for detailed analysis. The acquisition times were set so that for each sample, approximately the same total number of electrons were collected at both low and high threshold energies. The number of electrons, acquisition times, and the ratio of acquisition times are given in Table I.

As shown in Table I, to maintain the same number of collected electrons required an increase of the acquisition time by a factor of $\approx 125$ for diamond, $\approx 30$ for $\mathrm{Si}$, and $\approx 6$ for $\mathrm{GaN}$. The differences in this ratio of acquisition times may be attributed to the differences in the energy distribution of backscattered electrons for these materials. The differences between the energy distributions of electrons 


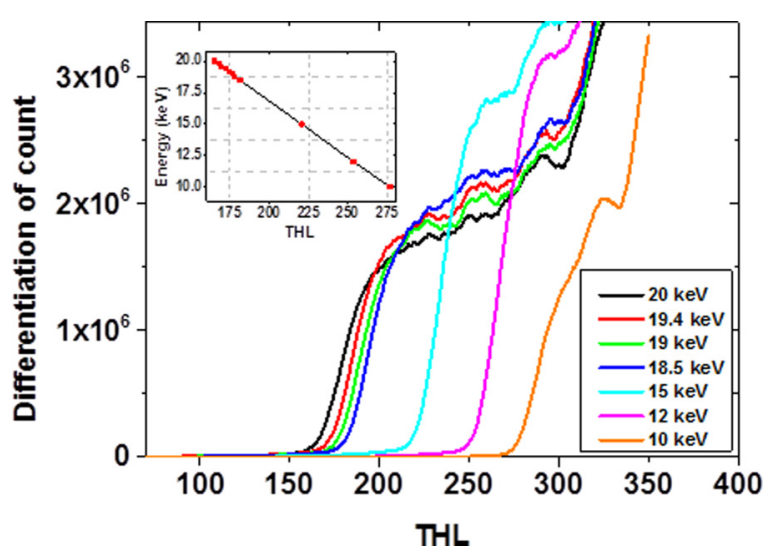

FIG. 5. (Color online) Threshold calibration using backscattered electrons showing the differentiation of the particle count as a function of the digital threshold value (THL). The inset at the top left shows the energy calibration line derived by extrapolating the intercept to zero of the curves plotted in the main graph.

backscattered from light, low-density materials and heavy, dense materials are illustrated in Fig. 6 which shows the Monte Carlo simulations of the backscattered electron energy spectra for $\mathrm{Si}$ (atomic weight 30, density $2330 \mathrm{~kg} \mathrm{~m}^{-3}$ ) and $\mathrm{Au}$ (atomic weight 197 , density $19300 \mathrm{~kg} \mathrm{~m}^{-3}$ ), respectively. The simulations use a differential inverse inelastic mean-free-path approach which has been found to predict correctly both the elastic peak at the energy of the primary beam [38], as well as the plasmon loss peaks observed in experiment [8].

The intensities have been scaled so that, for each element the total backscattered intensity from 10 to $20 \mathrm{keV}$ is taken as 1. We can see that Au has a relative elastic peak intensity of 0.055 while for $\mathrm{Si}$ it is 0.0045 . This means that for $\mathrm{Au}, 5.5 \%$ of the total $(10-20 \mathrm{keV})$ number of electrons is in the elastic peak alone, while for $\mathrm{Si}$ it is only around $0.5 \%$. In addition, for Au there is a greater intensity of electrons near the elastic peak than for Si. In general as illustrated in Fig. 6, for lighter, less dense materials the backscattered electrons have a much broader energy spectrum; that is, there are more electrons with higher energy loss, so fewer with energy close to that of the incident electron beam. Therefore for a light and low-density material, a longer acquisition time is required to acquire a highenergy-filtered EBSP pattern with a given number of electrons compared to that required for a heavy, dense material.

Simulations of EBSPs for diamond, $\mathrm{Si}$, and $\mathrm{GaN}$ using the dynamical diffraction theory approach [7] were carried out for

TABLE I. Comparison of acquisition times for diamond, silicon, and $\mathrm{GaN}$ samples with change in threshold energy.

\begin{tabular}{lcccc}
\hline \hline Specimen & $\begin{array}{c}\text { Threshold energy } \\
(\mathrm{keV})\end{array}$ & Count & Acq. time(s) & Ratio \\
\hline Diamond & 5.5 & $7.00 \times 10^{8}$ & 0.8 & $\approx 125$ \\
& 19.4 & $7.00 \times 10^{8}$ & 100 & \\
Silicon & 4.6 & $4.00 \times 10^{10}$ & 50 & $\approx 30$ \\
& 19.4 & $4.00 \times 10^{10}$ & 1482 & \\
GaN & 5.5 & $6.00 \times 10^{9}$ & 10 & $\approx 6$ \\
& 19.4 & $6.00 \times 10^{9}$ & 60 & \\
\hline \hline
\end{tabular}

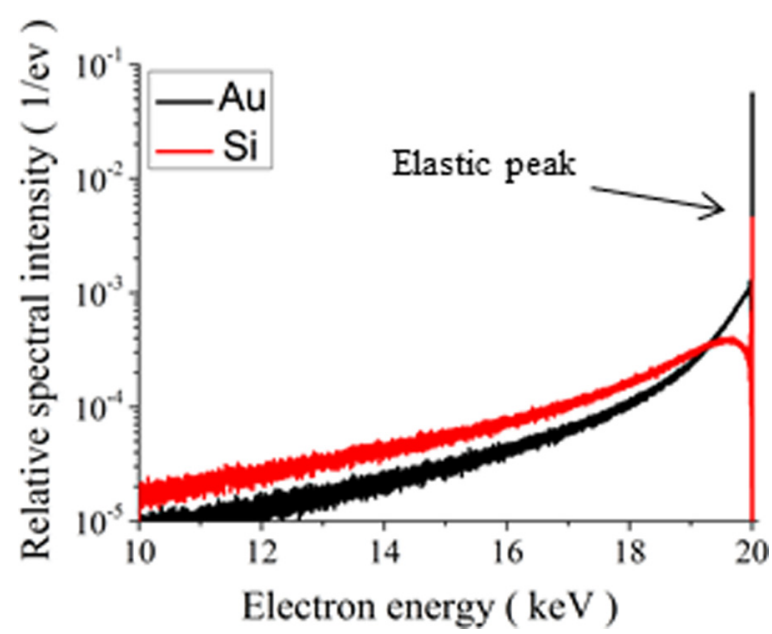

FIG. 6. (Color online) Monte Carlo simulations of the backscattered electron energy spectra for $\mathrm{Si}$ and $\mathrm{Au}$, respectively. The intensities have been scaled so that the backscattered intensity from 10 to $20 \mathrm{keV}$ is taken as 1 .

a range of single energies from 18 to $20 \mathrm{keV}$ at intervals of $500 \mathrm{eV}$. As the energy changes, subtle changes are observed in the EBSPs. For example, the intensity profile of the Kikuchi bands changes (this will be discussed in more detail later in this paper). By comparing the experimental patterns with the simulations, the simulation at an energy of $19.5 \mathrm{keV}$ provided the best match to the EBSPs acquired with high threshold energy, based on the normalized cross correlation coefficient. These are shown in Figs. 4(g)-4(i) for diamond, Si, and GaN, respectively.

The insets placed at the bottom right of each EBSP in Fig. 4 show the power spectrum of the EBSP obtained by performing a two-dimensional fast Fourier transform (FFT) on the pattern. These power spectra provide an indication of the global EBSP quality, that is, a measure of the detail, contrast, and sharpness of the diffraction pattern. A two-dimensional power spectrum is a two-dimensional histogram of the frequency intensity. The lower frequencies are located close to the center of the power spectrum; the frequencies increase with increasing distance from the center. In the power spectra of the EBSP, an increase of the higher-frequency components is observed for the EBSPs acquired at higher threshold energy, indicating that these EBSPs are of higher quality and thus contain more information. For example, HOLZ rings are observed in the high-energy-filtered EBSP from diamond [see Fig. 4(d)] - these are not observed in the low-energy-filtered EBSP. Higher-order Kikuchi bands can also be discerned. For GaN fine detail is observable in the HOLZ rings for the high-energy-filtered EBSP [see Fig. 4(f)].

To illustrate the effect of filtering on the observation of higher-order Kikuchi bands more clearly, Figs. 7(a) and 7(b) show "zoomed-in" images (from Fig. 4) of a region around the (220) band for both high- and low-energy-filtered EBSPs from Si. Figures 7(c) and 7(d) show this band isolated from the rest of the pattern through selecting the FFT wave-vector directions associated with this band in the FFT spectrum and taking the inverse FFT [39]. In the image derived from the high-energyfiltered EBSP [Fig. 7(c)], (220), (440), (660), and (880) bands 

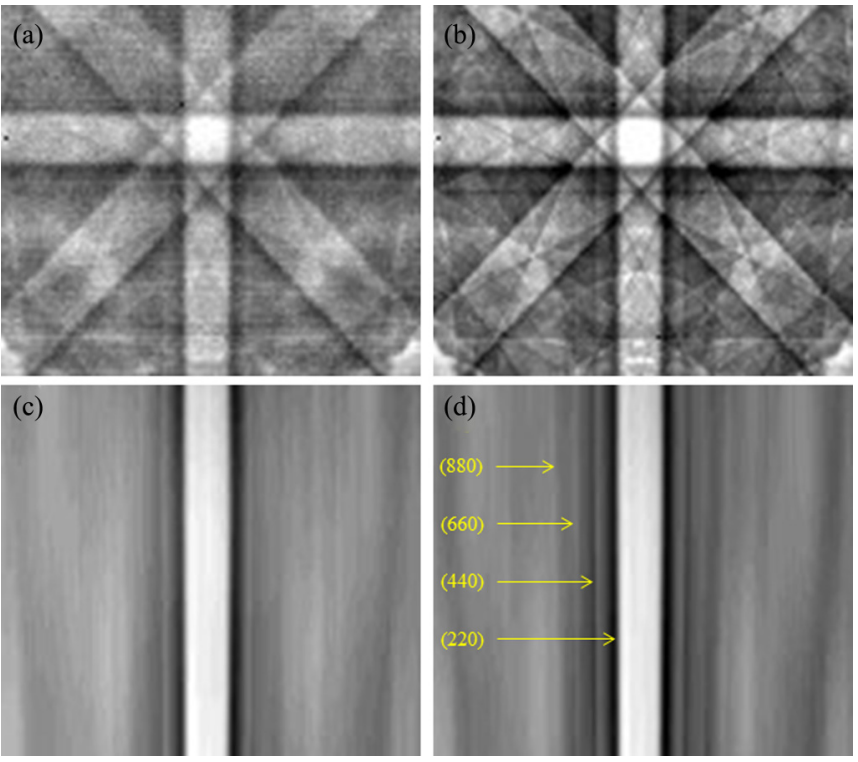

FIG. 7. (Color online) Zoomed-in regions from EBSPs from $\mathrm{Si}$ acquired with (a) low threshold and (b) high threshold energies; (c, d) show the extracted (220) and higher-order Kikuchi bands from the EBSPs shown in $(a, b)$, respectively.

can be discerned. The increase in the detail observed in the EBSPs is attributed to the removal of lower energy, i.e., highloss electrons which, as discussed in the Introduction, give rise to a featureless background. This background obscures the higher-order features; its removal results in EBSPs in which more detail is discernible.

To obtain a measure of the improvement in contrast in the EBSPs on energy filtering, the average contrast was calculated for a Kikuchi band profile for both as-acquired (i.e., not flat fielded) low- and high-energy-filtered EBSPs. The contrast is defined as

$$
C=\frac{\text { Maximum band intensity }- \text { Minimum band intensity }}{\text { Minimum band intensity }+ \text { Maximum band intensity }}
$$

Table II summarizes the results obtained for all samples.

Note that the greatest change in contrast, on acquiring a high-energy-filtered EBSP, is obtained for diamond. The absolute change in contrast depends on a number of factors including the quality of the sample surface and the position of the detector relative to the sample. The detector's performance will also vary with threshold energy; for example, at low threshold energy an electron may be counted by more than

TABLE II. Comparison of contrast for low- and high-energyfiltered EBSPs.

\begin{tabular}{lcccc}
\hline \hline Specimen & $\begin{array}{c}\text { Threshold energy } \\
(\mathrm{keV})\end{array}$ & Band & Contrast & Contrast ratio \\
\hline Diamond & 5.5 & $\{220\}$ & 0.037 & $\approx 4.0$ \\
Silicon & 19.4 & $\{220\}$ & 0.149 & \\
& 4.6 & $\{220\}$ & 0.0304 & $\approx 2.5$ \\
GaN & 19.4 & $\{220\}$ & 0.0744 & \\
& 5.5 & $\{1120\}$ & 0.074 & $\approx 2.1$ \\
\hline \hline
\end{tabular}

one pixel [30,40]; this effect will be eliminated at higher threshold energy. The largest change in contrast, however, was always obtained for the diamond sample, while the smallest change in contrast was always obtained for the GaN. This may be attributed to the difference in the energy distribution of backscattered electrons for these materials as discussed previously. For lighter, less dense materials the backscattered electrons have a much broader energy spectrum; that is, there are more electrons with higher energy loss. These electrons contribute to the diffuse background as discussed previously; thus removing these electrons from the EBSP significantly improves the contrast in the pattern. Initially for a given material, care was taken to acquire the same number of electrons for EBSPs recorded at both high and low threshold energies. However, subsequent measurements revealed that, as long as the number of electrons was high enough to obtain an EBSP, the contrast improvement on energy filtering was independent of acquisition time. This is expected since the intrinsic contrast and sharpness of the diffraction pattern should not depend on the counting statistics. High threshold images were acquired for acquisition times of $0.5 \mathrm{~s}$ which showed the same improvement in contrast.

Finally, to further investigate the subtle changes in the diffraction features on energy filtering, the detector was placed further away from the sample and EBSPs acquired with a smaller capture angle of $\approx 30^{\circ}$. Figure 8 shows EBSPs (flat fielded) acquired from silicon with an incident electron beam energy of $30 \mathrm{keV}$, a probe current of $\approx 2.5 \mathrm{nA}$, and an acquisition time of $\approx 100 \mathrm{~s}$. Figure 8 (a) shows an EBSP acquired with a low threshold energy, Fig. 8(b) shows an EBSP acquired at high threshold energy, and Fig. 8(c) shows the difference between (a) and (b) obtained by first flat fielding the raw EBSPs, normalizing the resultant images, and subtracting (b) from (a).

Note that the difference in the patterns results in asymmetric intensity features on the upper and lower sides of the nonvertical Kikuchi bands. This may be the result of differences in the contribution of inelastically scattered electrons to each of the differently energy-filtered EBSPs. Close examination of Fig. 8(a), the EBSP acquired with the low threshold energy, reveals that the intensity profile of the Kikuchi bands is asymmetric; that is, one side of a band is brighter and the other darker than would be the case for a symmetric intensity profile. This is highlighted by the line profile of band A as shown in Fig. 8(d). We believe this excess-deficiency effect is due to anisotropic scattering of inelastic electrons which is a result of the geometry of the measurement and the differential cross section for inelastic scattering [15].

This excess-deficiency effect is unexpectedly reduced in the high-energy threshold filtered EBSP shown in Fig. 8(b) [also see corresponding line profile of band A in Fig. 8(d)]. This could be attributed to fewer inelastically scattered electrons, with energy close to the elastic peak, contributing to the pattern. The strength of the excess-deficiency asymmetry depends on the relative orientation of the Kikuchi bands with respect to the incident beam direction. Bands running parallel to the incident beam direction should not show this effect as illustrated by the much smaller asymmetry observed for band B [see Fig. 8(e)]. The difference between the patterns acquired at high and low threshold energies as shown in Fig. 8(c) 

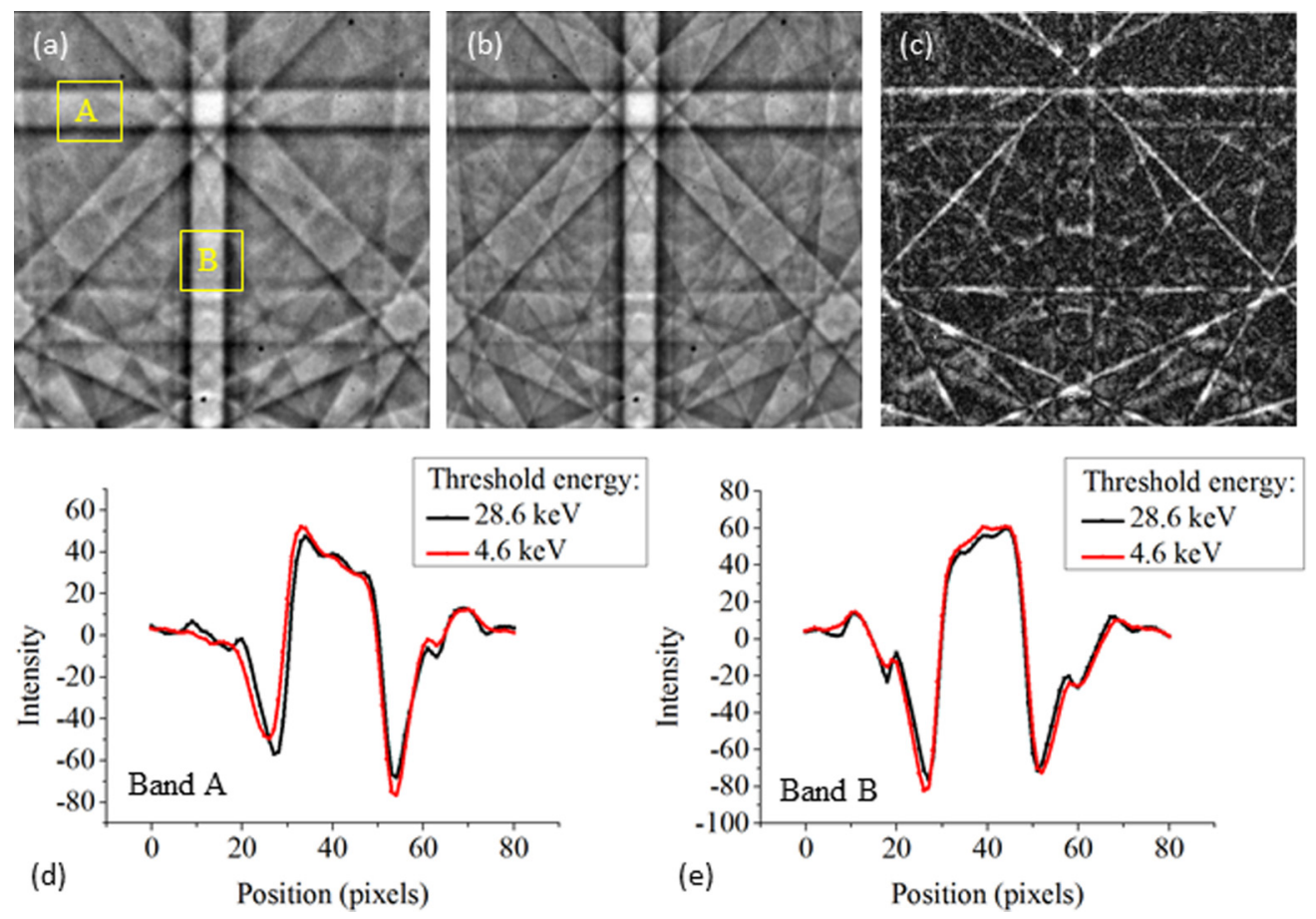

FIG. 8. (Color online) EBSPs from silicon for an incident beam energy of $30 \mathrm{keV}$, a probe current of $\approx 2.5 \mathrm{nA}$, an acquisition time of $\approx 100 \mathrm{~s}$, and a detector-to-specimen distance of $\approx 30 \mathrm{~mm}$ providing a capture angle of $\approx 30^{\circ}$. (a) Threshold energy of $4.6 \mathrm{keV}$, (b) threshold energy of $28.6 \mathrm{keV}$, (c) difference between (a) and (b), (d) mean Kikuchi band profile for band A in (a), (e) mean Kikuchi band profile for band $\mathrm{B}$ in (a), where the energy-dependent effect is much smaller than for band A.

reveals the energy dependence of the excess-deficiency effect across the EBSP. This effect is also visible in other features of the patterns, such as the edge of the HOLZ ring and the intersection of the Kikuchi bands, as indicated by the bright features (indicating large differences between the two patterns) in Fig. 8(c). To explain this unexpected observation requires a quantitative model describing the development of diffraction effects with energy loss. This involves the simultaneous treatment of the multiple inelastic scattering in the sample and dynamical diffraction effects, as has been outlined by Dudarev et al. using the density matrix formalism [41]. Our observations indicate that the details of the interrelated multiple inelastic scattering and dynamical diffraction are not completely understood and should thus provide a stimulus for an improved theoretical treatment which, while beyond our current capabilities, will be the focus of future work.

\section{SUMMARY}

To summarize, we have shown that digital energy filtering allows the acquistion of EBSPs exhibiting enhanced detail and contrast. The improvement in the quality of EBSPs is greatest for light, less dense, materials. This is attributable to the removal of electrons which contribute to a diffuse background and not to the diffraction pattern. This allows for example, the detection of high-order diffraction features. In addition comparison of low- and high-energy-filtered EBSPs implies that inelastically scattered electrons make a significant contribution to the appearance of the diffraction features in the EBSPs. The observed excess-deficiency effects are supressed on high-energy threshold filtering of the EBSPs. The ability to energy filter EBSPs will allow us to not only obtain a better understanding of the formation of EBSPs but also widen the application of EBSD to new materials and to new applications.

\section{ACKNOWLEDGMENTS}

We would like to thank Peter Parbrook, Tyndall National Institute, University College, Cork, Ireland for providing us with the GaN sample and Alexandre Tallaire, Laboratoire des Sciences des Procédés et des Matériaux LSPM-CNRS, Université Paris 13, France for providing the diamond sample. We would like to thank NPL for partial funding of Stefano Vespucci's Ph.D. studentship. This work was carried out with the support of EPSRC Grant No. EP/J015792/1. The experimental data presented in this paper is available at http://dx.doi.org/10.15129/7e864c9e-516c-465d-993db271f3698e1b or from the corresponding author.
[1] R. A. Schwarzer, D. P. Field, B. L. Adams, M. Kumar, and A. J. Schwartz, in Electron Backscatter Diffraction in Materials Science, edited byA. J. Schwartz, M. Kumar, B. L. Adams, and D. P. Field (Springer, New York, 2009), Vol. 2, Chap. 1, p. 1.
[2] J. Goldstein, D. E. Newbury, D. C. Joy, C. E. Lyman, P. Echlin, E. Lifshin, L. Sawyer, and J. R. Michael, in Scanning Electron Microscopy and X-ray Microanalysis (Springer, New York, 2003). 
[3] A. J. Wilkinson, J. Electron Microsc. 49, 299 (2000).

[4] A. P. Day, in Electron Backscatter Diffraction in Materials Science, edited by A. J. Schwartz, M. Kumar, B. L. Adams, and D. P. Field (Springer, New York, 2009), Vol. 2, Chap. 5, p. 65.

[5] A. J. Wilkinson, D. J. Dingley, and G. Meaden, in Electron Backscatter Diffraction in Materials Science, edited by A. J. Schwartz, M. Kumar, B. L. Adams, and D. P. Field (Springer, New York, 2009), Vol. 2, Chap. 17, p. 231.

[6] A. Winkelmann, in Electron Backscatter Diffraction in Materials Science, edited byA. J. Schwartz, M. Kumar, B. L. Adams, and D. P. Field (Springer, New York, 2009), Vol. 2, Chap. 2, p. 21.

[7] A. Winkelmann, C. Trager-Cowan, F. Sweeney, A. P. Day, and P. Parbrook, Ultramicroscopy 107, 414 (2007).

[8] A. Winkelmann, K. Aizel, and M. Vos, New J. Phys. 12, 053001 (2010).

[9] O. C. Wells, Scanning 21, 368 (1999).

[10] T. Matsukawa, R. Shimizu, and H. Hashimoto, J. Phys. D: Appl. Phys. 7, 695 (1974).

[11] G. E. Lloyd, Mineral. Mag. 51, 3 (1987).

[12] F. Yubero, N. Pauly, A. Dubus, and S. Tougaard, Phys. Rev. B 77, 245405 (2008).

[13] A. Winkelmann, F. Salvat-Pujol, and W. Werner, Microsc. Microanal. 19, 738 (2013).

[14] S. Zaefferer and N. N. Elhami, Acta Mater. 75, 20 (2014).

[15] A. Winkelmann, Ultramicroscopy 108, 1546 (2008).

[16] J. R. Michael and J. A. Eades, Ultramicroscopy 81, 67 (2000).

[17] A. Ichimiya and P. I. Cohen, Reflection High-Energy Electron Diffraction (Cambridge University Press, Cambridge, 2004).

[18] K. Z. Baba-Kishi, Ultramicroscopy 34, 205 (1990).

[19] P. G. Callahan and M. De Graef, Microsc. Microanal. 19, 1255 (2013).

[20] S. Villert, C. Maurice, C. Wyon, and R. Fortunier, J. Microsc. 233, 290 (2009).

[21] A. Winkelmann, J. Microsc. 239, 32 (2010).

[22] A. J. Eades, A. Deal, A. Bhattacharyya, and T. Hooghan, in Electron Backscatter Diffraction in Materials Science, edited byA. J. Schwartz, M. Kumar, B. L. Adams, and D. P. Field (Springer, New York, 2009), Vol. 2, Chap. 4, p. 53.
[23] A. Deal, T. Hooghan, and A. Eades, Ultramicroscopy 108, 116 (2008).

[24] A. Winkelmann and M. Vos, Ultramicroscopy 125, 66 (2013).

[25] X. Llopart, R. Ballabriga, M. Campbell, L. Tlustos, and W. Wong, Nucl. Instrum. Methods Phys. Res., Sect. A 581, 485 (2007).

[26] A. J. Wilkinson, G. Moldovan, T. B. Britton, A. Bewick, R. Clough, and A. I. Kirkland, Phys. Rev. Lett. 111, 065506 (2013).

[27] A. R. Faruqi and R. Henderson, Curr. Opin. Struct. Biol. 17, 549 (2007).

[28] http://medipix.web.cern.ch/medipix/

[29] X. Llopart, M. Campbell, R. Dinapoli, D. San Segundo, and E. Pernigotti, IEEE Trans. Nucl. Sci. 49, 2279 (2002).

[30] G. McMullan, D. M. Cattermole, S. Chen, R. Henderson, X. Llopart, C. Summerfield, L. Tlustos, and A. R. Farugi, Ultramicroscopy 107, 401 (2007).

[31] V. Kraus, M. Holik, J. Jakubek, M. Kroupa, P. Soukup, and Z. Vykydal, J. Instrum. 6, C01079 (2011).

[32] T. Holy, J. Jakubek, S. Pospisil, J. Uher, D. Vavrík, and Z. Vykydal, Nucl. Instrum. Methods Phys. Res., Sect. A 563, 254 (2006).

[33] http://aladdin.utef.cvut.cz/ofat/others/Pixelman/Pixelman.html

[34] L. Tlustos, R. Ballabriga, M. Campbell, E. Heijne, K. Kincade, X. Llopart, and P. Stejskal, IEEE Trans. Nucl. Sci. 53, 367 (2006).

[35] http://www.bruker.com/products/x-ray-diffraction-andelemental-analysis/eds-wds-ebsd-sem-micro-xrf-and-semmicro-ct/esprit-dynamics/overview.html.

[36] L. F. N. D. Carramate, F. Nachtrab, M. Firsching, A. L. M. Silva, A. M. da Silva, J. F. C. A. Veloso, and N. Uhlmann, J. Instrum. 8, C03022 (2013).

[37] S. Vespucci, Ph.D. thesis, Dept. of Physics, University of Strathclyde (to be published 2016).

[38] F. Salvat-Pujol, and W. S. Werner, Surf. Interface Anal. 45, 873 (2013).

[39] F. Ram, S. Zaefferer, and D. Raabe, J. Appl. Crystallogr. 47, 264 (2014).

[40] G. McMullan, S. Chen, R. Henderson, and A. R. Farugi, Ultramicroscopy 109, 1126 (2009).

[41] S. L. Dudarev, P. Rez, and M. J. Whelan, Phys. Rev. B 51, 3397 (1995). 